\title{
Neuronal activity in medial superior temporal area (MST) during memory-based smooth pursuit eye movements in monkeys
}

\author{
Sergei Kurkin · Teppei Akao • Natsuko Shichinohe • \\ Junko Fukushima $\cdot$ Kikuro Fukushima
}

Received: 2 July 2010 / Accepted: 2 August 2011 / Published online: 12 August 2011

(C) The Author(s) 2011. This article is published with open access at Springerlink.com

\begin{abstract}
We examined recently neuronal substrates for predictive pursuit using a memory-based smooth pursuit task that distinguishes the discharge related to memory of visual motion-direction from that related to movement preparation. We found that the supplementary eye fields (SEF) contain separate signals coding memory and assessment of visual motion-direction, decision not-to-pursue, and preparation for pursuit. Since medial superior temporal area (MST) is essential for visual motion processing and projects to SEF, we examined whether MST carried similar signals. We analyzed the discharge of 108 MSTd neurons responding to visual motion stimuli. The majority (69/ $108=64 \%$ ) were also modulated during smooth pursuit. However, in nearly all $(104 / 108=96 \%)$ of the MSTd neurons tested, there was no significant discharge modulation
\end{abstract}

S. Kurkin · T. Akao $\cdot$ N. Shichinohe $\cdot$ K. Fukushima

Department of Physiology, School of Medicine,

Hokkaido University, Sapporo, Japan

Present Address:

T. Akao

Department of Physiology, Asahikawa Medical College,

Midorigaoka, Asahikawa, Hokkaido 078-8510, Japan

N. Shichinohe

Department of Ophthalmology, School of Medicine,

Hokkaido University, Sapporo, Japan

J. Fukushima $\cdot$ K. Fukushima

Faculty of Health Sciences,

Hokkaido University, Sapporo, Japan

K. Fukushima ( $\square$ )

Clinical Brain Research Laboratory, Department of Neurology,

Toyokura Memorial Hall, Sapporo Yamanoue Hospital,

Yamanote 6-9-1-1, Nishiku, Sapporo 063-0006, Japan

e-mail: kikuro@med.hokudai.ac.jp during the delay periods that required memory of visual motion-direction or preparation for smooth pursuit or not-to-pursue. Only 4 neurons of the 108 (4\%) exhibited significantly higher discharge rates during the delay periods; however, their responses were non-directional and not instruction specific. Representative signals in the MSTd clearly differed from those in the SEF during memorybased smooth pursuit. MSTd neurons are unlikely to provide signals for memory of visual motion-direction or preparation for smooth pursuit eye movements.

Keywords MST $\cdot$ Visual motion $\cdot$ Memory $\cdot$ Movement preparation $\cdot$ Smooth pursuit $\cdot$ Monkey

\section{Introduction}

Smooth pursuit eye movements are essential to obtain accurate visual information about slowly moving objects. During smooth pursuit, response delays are compensated to maintain target images on the foveae; however, neuronal mechanisms of this predictive pursuit are still poorly understood (e.g., Becker and Fuchs 1985; Barnes and Asselman 1991; see Leigh and Zee 2006 for review). To examine neuronal substrates for predictive pursuit, the discharge related to movement preparation must be distinguished from the discharge related to processing of target motion signals or their memory (e.g., Assad and Maunsell 1995; Collins and Barnes 2005). Moreover, in daily life, there are many moving objects necessitating selection of a specific target, and for this, a decision of whether to pursue is also necessary.

To examine the neuronal substrates for these functions, we trained Japanese monkeys to perform a memory-based smooth pursuit task (Shichinohe et al. 2009). In this task, 
we used 2 cues, cue 1 for visual motion and cue 2 for decision to prepare to pursue (i.e., go) or not to pursue (i.e., no-go). Based on the memory of the visual motiondirection presented at cue 1 and the go/no-go instruction presented at cue 2 , monkeys had to select the correct pursuit direction or not pursue at all. We have shown that the SEF contains separate signals reflecting memory and assessment of visual motion-direction, the decision of notto-pursue during no-go trials, and movement preparation during go trials (Shichinohe et al. 2009). An important question is how these signals are generated in the SEF.

MST, an essential area for visual motion processing, contains at least two major subdivisions: the dorsomedial MST (MSTd) and ventrolateral MST (MSTl) (Desimone and Ungerleider 1986; see Leigh and Zee 2006 for a review). Most MSTd neurons have large visual receptive fields and strong directional selectivity for visual motion and discharge during, typically after the onset of, smooth pursuit eye movements (Saito et al. 1986; Komatsu and Wurtz 1988a; Newsome et al. 1988; Graziano et al. 1994; cf. Ferrera and Lisberger 1997). Celebrini and Newsome $(1994,1995)$ reported the involvement of MSTd in perceiving visual motion. Gu et al. $(2007,2008)$ suggested that MSTd neurons are involved in object motion perception by integrating eye and head movement-related signals (also Liu and Angelaki 2009). In contrast, MSTl contains visual tracking neurons that respond best to motion of small spots of light and discharge before the onset of smooth pursuit eye movements (Thier and Erickson 1992; Ferrera and Lisberger 1997; Dicke and Thier 1999; Ilg et al. 2004). Since MST, especially MSTd, sends direct projections to the SEF (Huerta and Kaas 1990), it is possible that the above SEF signals come from MSTd.

In the present study, we examined neuronal activity in MSTd during the memory-based smooth pursuit task (Shichinohe et al. 2009). If the SEF signals that code memory of visual motion-direction come from MSTd, we should observe signals in MSTd that are similar to those in the SEF. Some of our results have been presented in preliminary form (Kurkin et al. 2009).

\section{Materials and methods}

\section{General procedures}

Two monkeys (Macaca fuscata, Jl and Sh, 5-6 years old) were used. These monkeys were the same monkeys previously used for the analysis of SEF neuron discharge during memory-based smooth pursuit (Shichinohe et al. 2009). MST recordings were obtained during the same months while neuronal recording was made in the SEF. All procedures complied with the National Institutes of Health guidelines for the Care and Use of Animals. Our specific procedures were approved by the Animal Care and Use Committee of Hokkaido University School of Medicine. Our methods for animal preparation, training, recording, and data analysis are described elsewhere in detail (e.g., Fukushima et al. 2000; Shichinohe et al. 2009) and are briefly summarized here. Each monkey was sedated with ketamine hydrochloride $(5 \mathrm{mg} / \mathrm{kg}$, i.m.) and then anesthetized with sodium pentobarbital $(25 \mathrm{mg} / \mathrm{kg}$, i.p.). Additional anesthesia $(0.5-1.0 \%$ halothane mixed with $50 \%$ nitrous oxide and $50 \%$ oxygen) was administered as necessary. Under aseptic conditions, head holders were affixed to the skull of each monkey. Vertical and horizontal components of eye movements were recorded using a scleral search coil (Fuchs and Robinson 1966).

A recording chamber was stereotaxically implanted (center aimed at posterior $5 \mathrm{~mm}$ and lateral $15 \mathrm{~mm}$ ) on the skull to allow single-unit recording in MST (e.g., Komatsu and Wurtz 1988a, b; Newsome et al. 1988; Akao et al. 2005; Fujiwara et al. 2011). Analgesics (pentazocine, $0.2 \mathrm{mg} / \mathrm{kg}$ ) and antibiotics (flomoxef sodium, $50 \mathrm{mg} / \mathrm{kg}$ ) were administered postsurgically.

\section{Behavioral paradigms and recording procedures}

Each monkey was seated in a primate chair in darkness with the head firmly restrained, facing a 22-inch computer display (Mitsubishi, RDF 221S, $120 \mathrm{~Hz}$ ) placed $65 \mathrm{~cm}$ away from the eyes. Visual objects (spot and random-dot pattern, see below) were presented in the central $20^{\circ}$ by $20^{\circ}$ of the visual field. The task conditions are schematically illustrated in Fig. 1. A red stationary spot $\left(0.5^{\circ}\right.$ diameter $)$ appeared in the center, and the monkeys were required to fixate it (Fig. 1, 1. fixation). At cue 1, a random-dot pattern was presented (each $0.5^{\circ}$ spot, occupied $40 \%$ of the $20^{\circ} \times 20^{\circ}$ area, $\sim 600$ dots) and was moved along one of the 8 directions separated by $45^{\circ}$ at $10^{\circ} / \mathrm{s}$ for $0.5 \mathrm{~s}$ (Fig. 1 , 2. cue 1); horizontal (right or left), vertical (up or down), or 4 diagonal directions. Each dot in the pattern moved in the same direction (i.e., 100\% correlation, Newsome and Pare 1988). In successive trials, the direction of the moving pattern (e.g., right or left) was random but of equal frequency for each direction. The monkeys were required to remember the color of the pattern and the movement direction. After a delay (Fig. 1, 3. delay 1 of 1-4 s, typically $2 \mathrm{~s}$ ), a stationary pattern was presented as the second cue for $0.5 \mathrm{~s}$ (Fig. 1, 4. cue 2) (each $0.5^{\circ}$ spot, presented across $40 \%$ of the $20^{\circ} \times 20^{\circ}$ area, $\sim 600$ dots). If the color of cue 2 was the same as the cue 1 color, it instructed the monkeys to prepare to pursue a spot that would move in the direction instructed by cue 1 (i.e., go). If the cue 2 color differed from the cue 1 , it instructed the monkeys not to pursue (i.e., no-go) but to maintain fixation on a stationary spot. After the 


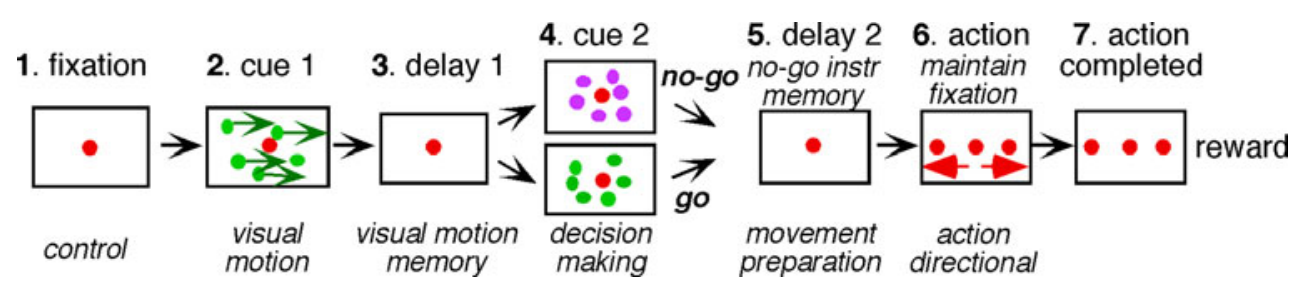

Fig. 1 The task conditions. For further explanation, see text

second delay (Fig. 1, 5. delay 2, typically $2 \mathrm{~s}$ ), the monkeys were required to perform the pursuit eye movement by selecting the correct spot (Fig. 1, 6. action). For this, the fixation spot remained stationary, but spawned 2 identical spots; one moved in the direction instructed by cue 1 and the other moved in the opposite direction at $10 \%$ s. The monkeys were required to respond correctly, either to pursue the correct spot or not to pursue (i.e., no-go) by remaining fixation of the stationary spot. The frequency of occurrence of the fixation condition was set at $24 \%$ of the trials, and in the remaining $76 \%$, the monkeys were required to pursue one of the 2 moving spots as described above.

Reward circuits compared the monkeys' eye position signals with the position signals of the stationary spot during the initial fixation, cue 1 , cue 2 , and two delay periods and with the correct target spot during the action period (Fig. 1). If the monkeys' gaze was within the error window of $\pm 2^{\circ}$, apple juice was automatically delivered to the animal at the end of each trial (Fig. 1, reward). If the monkeys' gaze was outside the error window, the trial was aborted and was restarted. The monkeys were also trained to perform the task while cue 1 and cue 2 colors were changed. Typically, we prepared 5 sets of different colored dots, and each set was presented randomly within the block. The monkeys were trained to perform this task over several months to a year. By the time, we started MST recordings, the error rate was less than $10 \%$.

Extracellular recordings were made in the 2 monkeys. Once task-related neurons in MST were isolated (See "Data analysis"), we determined preferred directions for their responses by moving cue 1 along different directions using 100\% correlation (Newsome and Pare 1988). To estimate the receptive field size of individual MST neurons and to examine whether the receptive field included the ipsilateral hemifield, we also used smaller cue 1 size (e.g., $5^{\circ} \times 5^{\circ}$, or $10^{\circ} \times 10^{\circ}$ ) and shifted the center of the cue 1 by $12^{\circ}$ from the center of the display to left, right, up, or down.

For cue 1, we also moved each dot randomly using $0 \%$ correlation in a block of trials (Newsome and Pare 1988) as tested for SEF neurons. If the color of cue 2 was the same as cue 1, it instructed $g o$ and the monkey followed one of the 2 moving spots during the action period. If the color of cue 2 differed from that of cue 1, it instructed no-go. The $0 \%$ correlation was used to let the monkeys choose pursuit direction themselves during action as used in our previous studies (Shichinohe et al. 2009) and to examine whether discharge of MST neurons during the delay 1 and delay 2 was correlated with the direction of subsequent pursuit eye movements or no-go instruction as observed in SEF neurons.

Data analysis

To analyze the discharge of each neuron, traces were aligned on the onset of cue 1. Eye position, target position, and neuronal discharge were sorted by correct direction instructed by cue 1 and cue 2. Trials for $g o$ and no-go were sorted separately. Mean discharge rates of individual neurons during each period (e.g., Fig. 2a, periods 2-7) were measured and compared as the mean $( \pm \mathrm{SD})$ rate of each period versus the mean discharge rate $( \pm \mathrm{SD})$ during the initial fixation (e.g., Fig. 2a, period 1), which acted as a control for each neuron. We defined significant differences as those having a $P$-value $<0.05$ using Student's $t$-test with the Bonferroni correction for multiple comparisons (Shichinohe et al. 2009). Neurons that exhibited significant modulation during our task were defined as task-related neurons. The monkeys occasionally made small eye movements during the delay periods (e.g., Fig. 2a1). Some were blinks. These eye movements did not contribute to the observed neuronal responses.

Latencies of neuronal responses to cue 1 visual motion were determined as the time at which the mean discharge rate exceeded $2 \mathrm{SD}$ of the control fixation rate. Latencies to the onset of target motion during the action period of go trials along the preferred directions were determined as the time at which the mean discharge rate exceeded $2 \mathrm{SD}$ of the discharge during delay 2 .

\section{Histological procedures}

Near the conclusion of recordings in the 2 monkeys, some of the recording sites were marked by passing current ( $50 \mu \mathrm{A}$ for $30 \mathrm{~s}$ ) through the tip of an iron-plated tipped, tungsten electrode. After recording was completed, the 
Fig. 2 Discharge of a representative MST neuron. a1-2, go trials when leftward (a1) and rightward (a2) visual motion was applied as cue 1. b1-2, no-go trials when leftward (b1) and rightward (b2) visual motion was applied as cue 1 . $\mathbf{a} 3$ and $\mathbf{b 3}$ compare mean discharge rates during leftward (thick)/rightward (thin) cue 1 visual motion for $g o$ trials (a3) and no-go trials (b3)
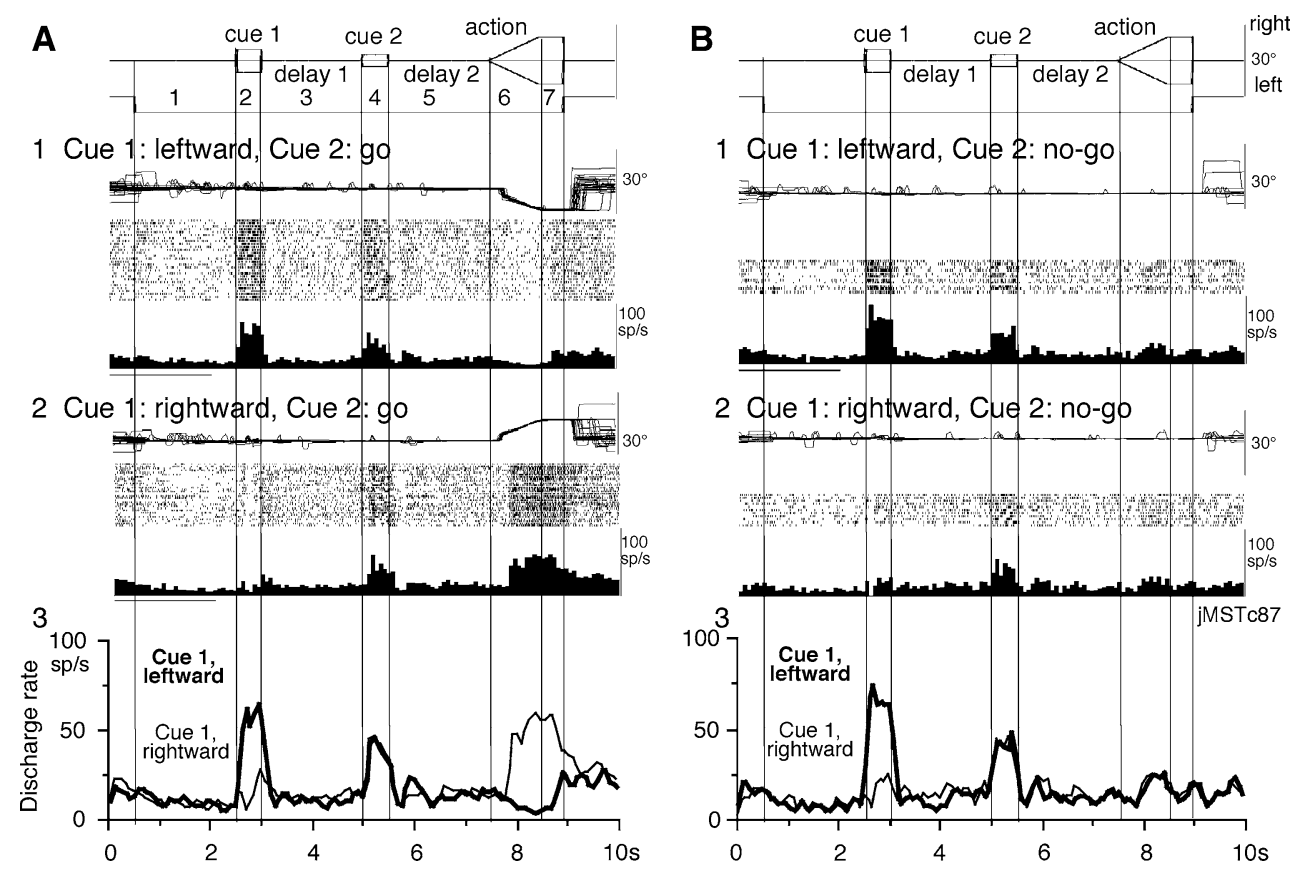

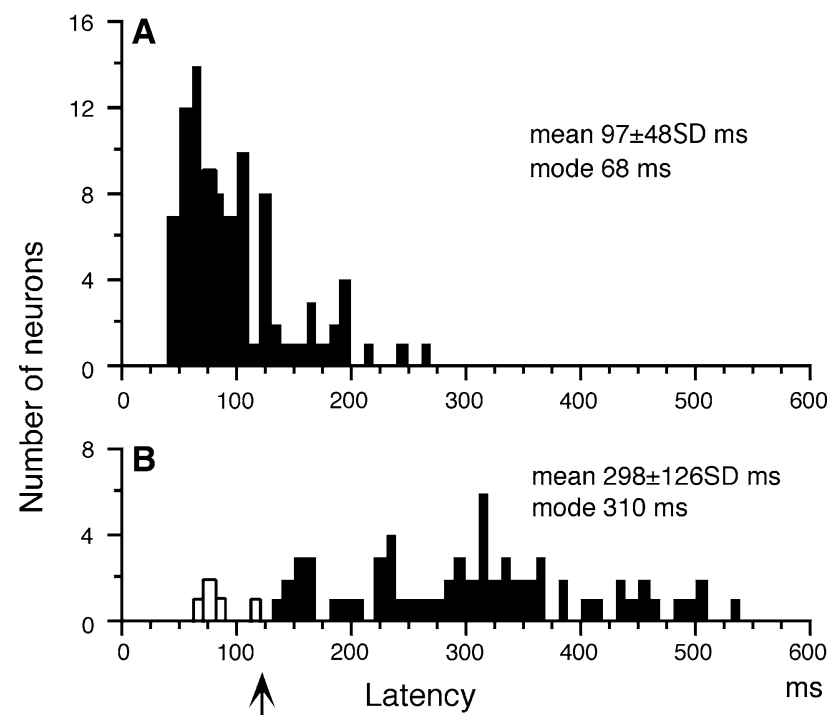

Fig. 3 Latencies of neuronal responses cue 1 visual motion (a) and spot motion during smooth pursuit of $g o$ trials during the action period (b). Open bars in b indicate neurons that discharged before the onset of smooth pursuit eye movements. Upward arrow in $\mathbf{b}$ indicates mean latency of smooth pursuit eye movements during the action period

monkeys were deeply anesthetized with pentobarbital sodium $(50 \mathrm{mg} / \mathrm{kg}$, i.p.) and perfused with physiological saline followed by $3.5 \%$ formalin. After histological fixation, coronal sections were cut at $100 \mu \mathrm{m}$ thickness on a freezing microtome. These sections were stained using the Nissl method, and the recording sites were verified microscopically as previously described (Akao et al. 2005; Fujiwara et al. 2011).

\section{Results}

Discharge of task-related neurons in MST

We recorded a total of 121 task-related neurons that exhibited significant modulation during our task (See "Data analysis"). Of these, 13 neurons did not show clear modulation during cue 1 visual motion; they were modulated mostly during the action period with or without modulation during delay periods. None of the 13 neurons exhibited directionspecific or instruction-specific discharge during delay periods. The remaining 108 neurons responded to cue 1 visual motion (73 from monkey Jl and 35 from monkey Sh). We performed further analysis for the 108 neurons. Discharge characteristics of neurons recorded in the 2 monkeys were similar. Of the 108, 98 neurons exhibited directional responses to cue 1 , and 10 neurons were non-directional. The majority of them $(69 / 108=64 \%)$ were also modulated during smooth pursuit eye movements during the action period. Preferred directions of visual motion responses and pursuit responses were similar in 33 neurons but were opposite in 36 neurons. All of these neurons showed strong directional selectivity, and the neurons that responded to cue 1 had large visual receptive fields, and in many of them, the ipsilateral hemifield was included.

Figure 2 illustrates the discharge of a representative neuron during go (Fig. 2a) and no-go trials (Fig. 2b). This neuron clearly showed a directional response to cue 1 when the pattern moved leftwards both during go trials (Fig. 2a1 vs. a2) and no-go trials (Fig. 2 b1 vs. b2) with similar response magnitudes (Fig. 2a1 vs. b1). Visual responses to cue 2 
were also weakly observed during both go and no-go trials (Fig. 2a1 vs. a2; b1 vs. b2) with similar magnitudes. During rightward pursuit during the action period, this neuron exhibited robust discharge that occurred after the onset of pursuit eye movements (Fig. 2a2), while during leftward pursuit, the discharge rate decreased (Fig. 2a1). During the action period of no-go trials, it exhibited only a weak response most probably reflecting a visual response to spot motion (Fig. 2b1, b2).

Latencies of neuronal responses to cue 1 visual motion along the preferred direction for each neuron and spot motion during the action period (i.e., smooth pursuit) are summarized in Fig. 3. In response to cue 1 visual motion, the modal value was $68 \mathrm{~ms}$ and the mean latency was $97 \mathrm{~ms}$ (Fig. 3a). In response to smooth pursuit of the action period during go trials, the modal value was $310 \mathrm{~ms}$ and the mean latency was $298 \mathrm{~ms}$ (Fig. 3b). Only 5 neurons (7\%) discharged before the onset of pursuit eye movements (Fig. 3b: open bars). The remaining neurons tested discharged after the onset of smooth pursuit eye movements (Fig. 3b, arrow). These response properties are typical of neurons in MSTd (e.g., Komatsu and Wurtz 1988a) and were also similar to the discharge properties of MSTd neurons in previous studies in this laboratory (Akao et al. 2005; Fujiwara et al. 2011).

The memory-based smooth pursuit task separates the neuronal discharge related to the memory of visual motiondirection from the discharge related to preparation for pursuit eye movements (Fig. 1; Shichinohe et al. 2009). Figure 2a3 superimposes the mean discharge rates of the representative neuron when the monkey performed rightward pursuit and leftward pursuit based on the memory of visual motion-direction presented at cue 1 (thin vs. thick). Similarly, Fig. $2 \mathrm{~b} 3$ superimposes mean discharge rates during no-go trials when the direction of cue 1 visual motion was rightward and leftward (thin vs. thick).

If this neuron coded memory of visual motion-direction, the discharge rate during delay 1 should have been significantly different from the control discharge rate and the two mean traces (thin vs. thick) should also have been significantly different as we found in visual memory neurons and visual memory + movement preparation neurons in the SEF (Shichinohe et al. 2009). However, during delay 1, there was no significant difference either in the modulation (vs. control) or in the discharge rate between the two traces (thin vs. thick Fig. 2a3 and b3).

If this neuron coded movement preparation or no-go, delay 2 discharge should have shown directional modulation or no-go instruction-specific discharge modulation; however, no such modulation occurred during go trials during delay 2 (Fig. 2a3, thin vs. thick) and no-go trials (Fig. 2b3, thin/thick vs. control). None of the 5 neurons that discharged before the onset of smooth pursuit eye movements (Fig. 3b, open bars) exhibited direction-specific discharge modulation during delay 2.

Virtually all of the 108 neurons tested $(104 / 108=96 \%)$ showed no significant discharge modulation during delay 1 or delay 2 during go trials and no-go trials (e.g., Fig. 2). Only 4 of the $108(4 \%)$ exhibited maintained cue 1 discharge during delay 1 during both go trials and no-go trials. These 4 neurons were also modulated during smooth pursuit eye movements during the action period of go trials. However, the delay 1 response was non-directional. Figure 4a shows an example neuron discharge that responded to leftward visual motion during cue 1 and discharged during rightward pursuit during action (Fig. 4a1, a2). The discharge rate during delay 1 was clearly higher than that during control fixation. However, as shown in Fig. 4a3, its discharge was not directional during delay 1 or delay 2 (thin vs. thick).

\section{Discharge modulation during $0 \%$ correlation at cue 1}

During 0\% correlation, cue 1 does not provide the necessary information about the direction of visual motion (Newsome and Pare 1988). As reported earlier (Shichinohe et al. 2009), our monkeys pursued one of the two moving spots randomly with nearly equal probability during the action period during go trials (See also "Materials and methods"). Previous studies indicate that the delay 1 activity of visual memory + movement preparation neurons in the SEF covaried with both the delay 2 activity and the monkeys' choice for final pursuit eye movement direction (Shichinohe et al. 2009), reflecting the monkeys' assessment of visual motion-direction (delay 1) and choice of pursuit direction preparation (delay 2). In the present study, we tested whether MSTd neurons that showed delay 1 response during $100 \%$ correlation at cue 1 exhibited a directional response during delay 1 correlated with the monkey's choice of pursuit direction during $0 \%$ correlation at cue 1 .

We sorted individual trials based on the monkeys' choice of either the preferred direction during the action period or the anti-preferred direction for each neuron (tested by $100 \%$ correlation). Figure 4 a shows discharge of the example neuron shown in Fig. 4a when the monkey performed leftward pursuit (Fig. 4b1) and rightward pursuit (Fig. 4b2) during 0\% correlation at cue 1. Discharge modulation during delay 1 was virtually identical for these two pursuit directions (Fig. 4b1 vs. b2). Although the delay 1 activity was significantly higher than the control discharge rate, there was no directional selectivity correlated with the pursuit direction during the action periods. This is clearly shown by the superimposed mean discharge rates in Fig. $4 \mathrm{~b} 3$ (delay 1 , thin vs. thick). The delay 2 activity of this neuron was also significantly higher than the control discharge rate but without directional selectivity (Fig. 4b3, thin vs. thick). 
Fig. 4 Discharge of an MST neuron that exhibited significantly higher discharge rates during delay periods. $\mathbf{a}$ and b compare discharge modulation during $g o$ trials when cue 1 was presented as $100 \%$ correlation (a) and $0 \%$ correlation (b). a1-2, cue 1 was leftward (a1) and rightward (a2). b1-2, monkey's choice for pursuit direction was leftward (b1) and rightward (b2). $\mathbf{a} 3$ and b3 compare mean discharge rates for a1-2 and b1-2 as indicated

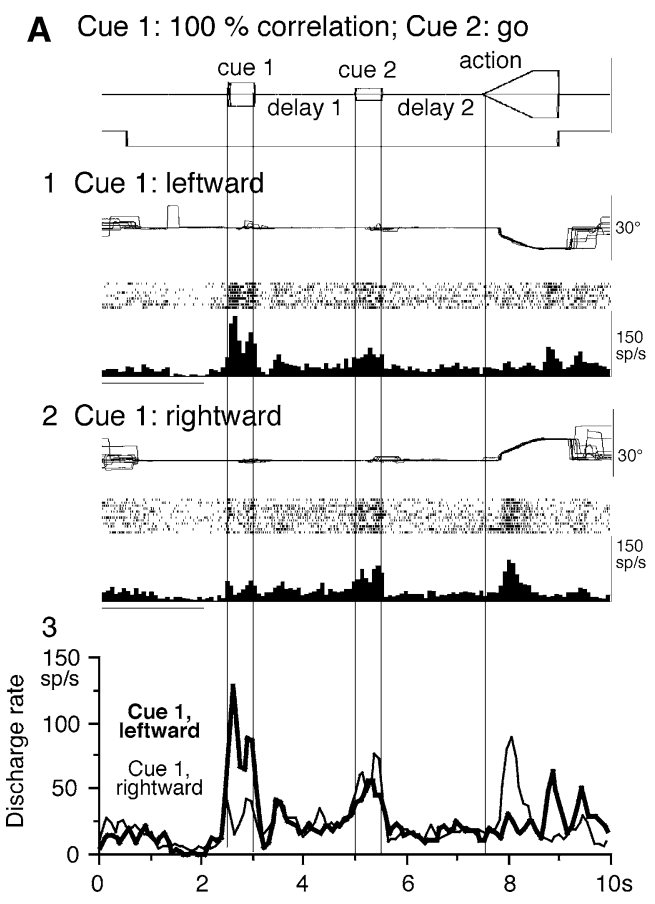

B Cue 1:0\% correlation; Cue 2: go

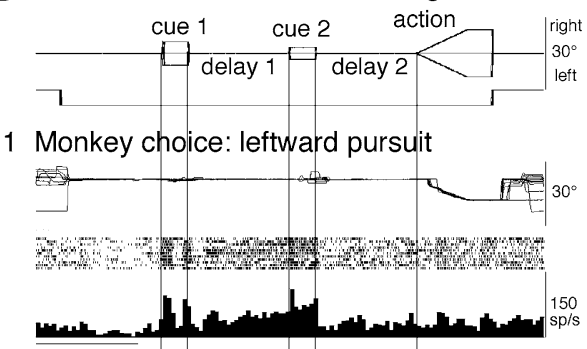

2 Monkey choice: rightward pursuit

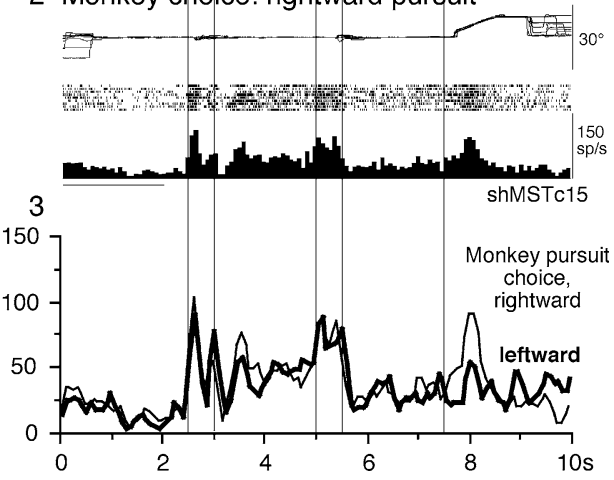

Figure 5a shows the time course of mean discharge rate of 10 MST neurons in which 0 and $100 \%$ correlation was tested at cue 1 . Cue 1 responses during $100 \%$ correlation were clearly directional (Fig. 5a1), but discharge during delay 1 and delay 2 was virtually identical; $0 \%$ correlation at cue 1 (Fig. 5a2) resulted in virtually identical responses during cue 1 , delay 1 , and delay 2 even though these neurons clearly showed directional responses during the action period based on the monkeys' choice of preferred- and antipreferred direction of each neuron during the action period (Fig. 5a1, a2).

For comparison, mean discharge rates of SEF visual memory + movement preparation neurons $(n=10)$ are shown in Fig. 5b during 100 and $0 \%$ correlation at cue 1 during go trials from previous studies (Shichinohe et al. 2009). In both 100 and $0 \%$ correlation conditions (Fig. 5 b1 and b2), these SEF neurons exhibited directional delay 1 and delay 2 responses. These results indicate that the MST tested neurons did not code directional signals during delay periods, although their discharge accurately reflected visual motion and/or visual stimuli applied on the retina (See "Discussion").

In both monkeys, recording tracks passed through the rostral bank of the superior temporal sulcus within or in close vicinity of MSTd, similar to the recording locations reported earlier (Akao et al. 2005; Fujiwara et al. 2011). The 4 neurons that exhibited maintained cue 1 discharge during delay 1 during both go trials and no-go trials (Fig. 4) were recorded intermixed with other neurons that did not exhibit such discharge.

\section{Discussion}

In the memory-based smooth pursuit task, we used 2 cues: cue 1 for visual motion and cue 2 for decision to prepare to pursue (i.e., go) or not to pursue (i.e., no-go). Based on the memory of the visual motion-direction presented at cue 1 and the go/no-go instruction presented at cue 2, our monkeys had to select the correct pursuit direction or not pursue at all. Our results indicate that none of 108 task-related MSTd neurons tested exhibited directional delay 1 or delay 2 activity or delay 2 activity specific to no-go instruction (e.g., Figs. 3, 4, 5a). Comparison of discharge modulation of MST neurons with SEF visual memory + movement preparation neurons in previous studies (Fig. 5a vs. b) indicates a clear difference in signals represented in neurons in the two areas during memory-based smooth pursuit (Shichinohe et al. 2009).

In the present study, we tested the possibility that MSTd sends signals coding memory and assessment of visual motion-direction to the SEF for the following reasons: (1) MSTd is involved in visual motion processing (Saito et al. 1986; Dürsteler and Wurtz 1988; Komatsu and Wurtz 1988a,b; Newsome et al. 1988; Graziano et al. 1994) and has also been suggested to be involved in perception and/or memory of visual motion (Celebrini and Newsome 1994, 1995; Britten and Wezel 1998, 2002; Kawawaki et al. 2006; Gu et al. 2007, 2008; Liu and Angelaki 2009); and (2) MST, especially MSTd, sends direct projections to the SEF (see Fig. 13P, Q, R of Huerta and Kaas 1990). Our negative results suggest that, unlike visual memory + 


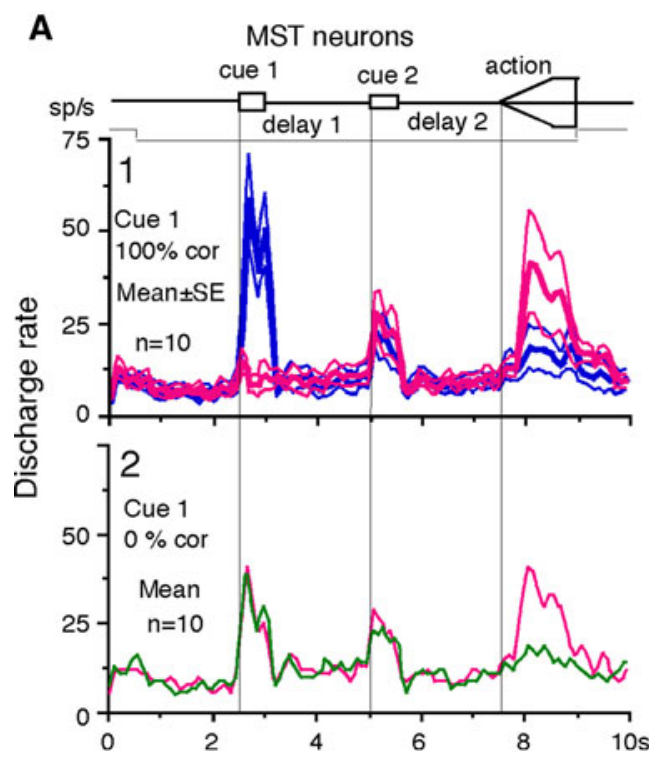

Fig. 5 Mean discharge rates of MST neurons during go trials when cue 1 was presented as 100 and $0 \%$ correlation and comparison with SEF visual memory + movement preparation neurons. a1, blue and pink traces compare mean $( \pm \mathrm{SE})$ discharge rates of $10 \mathrm{MST}$ neurons in their preferred directions (blue) and anti-preferred directions (pink) at cue 1 when cue 1 was $100 \%$ correlation. a2, green and pink traces compare mean discharge rates of the same 10 MST neurons based on whether the monkeys pursued in the preferred directions of individual neurons during action period (pink) or anti-preferred directions (green) when cue 1 was $0 \%$ correlation. b1 plots mean $( \pm \mathrm{SE})$ discharge rates

movement preparation neurons in the SEF, at least the MSTd neurons we tested are not directly involved in perceiving visual motion-direction or movement preparation in our task conditions (Fig. 5a vs. b). Our results are consistent with a previous report showing that single MST neuronal activity is dissociated from a monkeys' behavioral choice in a two-alternative, forced choice-task using optic flow information (Heuer and Britten 2004).

Of note is that we observed significantly higher discharge rates during delay 1 and delay 2 in a few MSTd neurons $(4 / 108=4 \%)$; however, their discharge during cue 1 at $0 \%$ correlation condition and during delay 1 was not directional (Figs. 4a, b, 5a). These results imply 2 points as follows: (1) MSTd neuronal discharge during cue 1 accurately reflected visual motion and/or visual stimuli applied on the retina, which is important for further conversion of visual motion signals. (2) But, unlike visual memory + movement preparation neurons in the SEF (Fig. 5b), MSTd neurons did not provide useful directional information during delay 1 or delay 2 to prepare for pursuit directions (Figs. 4b, 5a). We think that their activity during delay periods (Figs. 4b, 5a) most probably reflected an effect of attention (e.g., Recanzone and Wurtz 2000).

By manipulating visual inputs during pursuit eye movements, Newsome et al. (1988) demonstrated that the

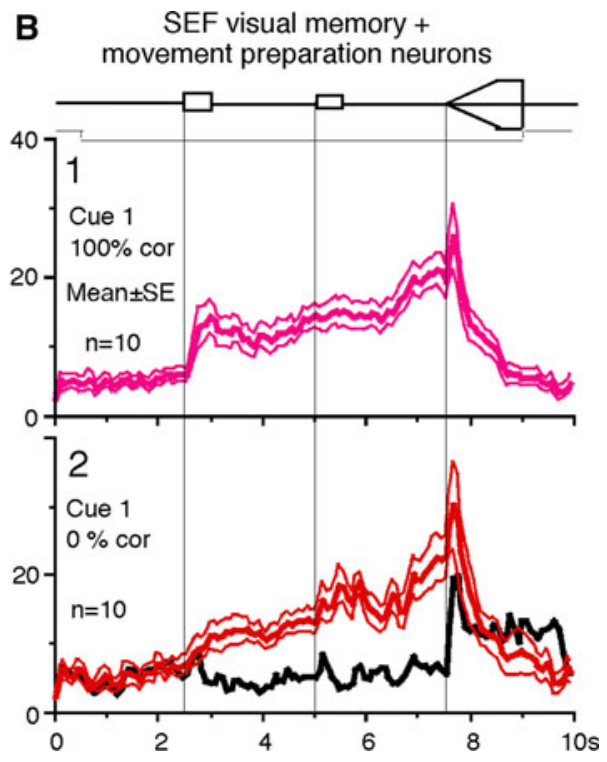

of $10 \mathrm{SEF}$ visual memory + movement preparation neurons in their preferred directions during delay 2 when cue 1 was $100 \%$ correlation. Red traces in $\mathbf{b} 2$ plot mean ( \pm SE) discharge rates of the same $10 \mathrm{SEF}$ neurons when the monkeys pursued in the preferred directions of individual neurons during delay 2 when cue 1 was $0 \%$ correlation. Black trace in $\mathbf{b 2}$ is mean discharge rate of the same 10 SEF neurons in their anti-preferred directions during delay 2 when cue 1 was $0 \%$ correlation. Traces in $\mathbf{b 2}$ are reanalyzed data obtained in the experiment published in Shichinohe et al. (2009)

extraretinal, pursuit response of MSTd neurons begins at least $50 \mathrm{~ms}$ after onset of the smooth pursuit eye movements. They suggested that this response most likely derives from corollary discharge mechanisms and that MST plays a role in generating the motor signals responsible for the maintenance of ongoing pursuit. The present results showing lack of movement preparation signals (Figs. 2, 5a) and late onset of MSTd neuron modulation (Fig. 3b) during the action period of $g o$ trials are consistent with their observation (Newsome et al. 1988).

It is possible that visual motion-direction information sent to the SEF from MSTd is further processed in the SEF to create the memory and assessment of visual motiondirection (Shichinohe et al. 2009; also See "Discussion" of Fukushima et al. 2011). However, we do not exclude the possibility that there may be another type of neurons coding memory and assessment of visual motion-direction in the MSTd that we missed (Ferrera and Lisberger (1997). Also, we did not record in MSTl where visual tracking neurons are located (e.g., Thier and Erickson 1992; Bremmer et al. 1997, 1999; Dicke and Thier 1999; Ilg et al. 2004), and the possibility remains that MSTl neurons may have signals similar to the SEF. Furthermore, our results do not preclude the possibility that signals similar to the SEF may be found in the visual posterior sylvian area (VPS, Guldin and 
Grüsser 1998; Dicke et al. 2008) and the ventral intraparietal cortex (VIP, Colby et al. 1993). In preliminary studies, we recorded 10 VIP neurons in the same monkeys; however, their discharge characteristics were similar to MSTd neurons in the present study and none of them exhibited directional delay 1 or delay 2 activity or no-go instructionspecific delay 2 activity (Akao et al. unpublished observations). Dicke et al. (2008) reported that percept-related neurons were more frequent in VPS than in V1 and MT/ MST. Single neuron recording in MSTl, VPS, and VIP would be necessary during memory-based smooth pursuit eye movements in future studies to test whether these areas signal memory of visual motion-direction.

In summary, our results indicate that MSTd neurons signal visual motion accurately, but they are unlikely to provide signals for memory of visual motion-direction or preparation for pursuit eye movement during memorybased smooth pursuit eye movements.

Acknowledgments We thank Dr. Peter M. Olley of Sappporo Medical University for his valuable comments on the manuscript and Mr. Motoyuki Kanashima for technical assistance. This research was supported by Grant-in-Aid for Scientific Research on Priority Areas (System study on higher-order brain functions) (17022001) and (C) (20500351) from the Ministry of Education, Culture, Sports, Science and Technology of Japan.

Conflict of interest The authors declare that they have no conflict of interest.

Open Access This article is distributed under the terms of the Creative Commons Attribution Noncommercial License which permits any noncommercial use, distribution, and reproduction in any medium, provided the original author(s) and source are credited.

\section{References}

Akao T, Mustari J, Fukushima J, Kurkin S, Fukushima K (2005) Discharge characteristics of pursuit neurons in MST during vergence eye movements. J Neurophysiol 93:2415-2434

Assad JA, Maunsell JHR (1995) Neuronal correlates of inferred motion in primate posterior parietal cortex. Nature 37:518-521

Barnes GR, Asselman PT (1991) The mechanism of prediction in human smooth pursuit eye movements. J Physiol (Lond) 439:439-461

Becker W, Fuchs AF (1985) Prediction in the oculomotor system: smooth pursuit during transient disappearance of a visual target. Exp Brain Res 57:562-575

Bremmer F, Ilg UJ, Thiele A, Distler C, Hoffmann KP (1997) Eye position effects in monkey cortex. I. Visual and pursuit-related activity in extrastriate areas MT and MST. J Neurophysiol 77:944-961

Bremmer F, Kubischik M, Pekel M, Lappe M, Hoffmann KP (1999) Linear vestibular self-motion signals in monkey medial superior temporal area. Ann NY Acad Sci 871:272-281

Britten KH, van Wezel RJ (2002) Area MST and heading perception in Macaque monkeys. Cereb Cortex 12:692-701

Britten KH, Wezel RJ (1998) Electrical microstimulation of cortical area MST biases heading perception in monkeys. Nat Neurosci $1: 59-63$
Celebrini S, Newsome WT (1994) Neuronal and psychophysical sensitivity to motion signals in extrastriate area MST of the macaque monkey. J Neurosci 14:4109-4124

Celebrini S, Newsome WT (1995) Microstimulation of extrastriate area MST influences performance on a direction discrimination task. J Neurophysiol 73:437-448

Colby CL, Duhamel L, Goldberg ME (1993) Ventral intraparietal area of macaque: anatomic location and visual response properties. J Neurophysiol 69:902-914

Collins CJ, Barnes GR (2005) Scaling of smooth anticipatory eye velocity in response to sequences of discrete target movements in humans. Exp Brain Res 167:404-413

Desimone R, Ungerleider LG (1986) Multiple visual areas in the superior temporal sulcus of the macaque. J Comp Neurol 248:164-189

Dicke P, Thier P (1999) The role of cortical area MST in a model of combined smooth eye-head pursuit. Biol Cybern 80:71-84

Dicke P, Chakraborty S, Thier P (2008) Neuronal correlates of perceptual stability during eye movements. Eur J Neurosci 27:991-1002

Dürsteler MR, Wurtz RH (1988) Pursuit and optokinetic deficits following chemical lesions of cortical areas MT and MST. J Neurophysiol 60:940-965

Ferrera VP, Lisberger SG (1997) Neuronal responses in visual areas MT and MST during smooth pursuit target selection. J Neurophysiol 78:1433-1446

Fuchs AF, Robinson DA (1966) A method for measuring horizontal and vertical eye movements chronically in the monkey. J Appl Physiol 21:1068-1070

Fujiwara K, Akao T, Kurkin S, Fukushima K (2011) Activity of pursuit-related neurons in medial superior temporal area (MST) during static roll-tilt. Cereb Cortex 21:155-165

Fukushima K, Sato T, Fukushima J, Shinmei Y, Kaneko CRS (2000) Activity of smooth pursuit-related neurons in the monkey periarcuate cortex during pursuit and passive whole-body rotation. J Neurophysiol 83:563-587

Fukushima J, Akao T, Shichinohe N, Kurkin S, Kaneko CRS, Fukushima K (2011) Neuronal activity in the caudal frontal eye fields of monkeys during memory-based smooth-pursuit eye movements: comparison with the supplementary eye fields. Cereb Cortex 21:1910-1924

Graziano MS, Andersen RA, Snowden RJ (1994) Tuning of MST neurons to spiral motions. J Neurosci 14:54-67

Gu Y, DeAngelis GC, Angelaki DE (2007) A functional link between area MSTd and heading perception based on vestibular signals. Nat Neurosci 10:1038-1047

Gu Y, Angelaki DE, DeAngelis GC (2008) Neural correlates of multisensory cue integration in macaque MSTd. Nat Neurosci 11:1201-1210

Guldin WO, Grüsser OJ (1998) Is there a vestibular cortex? Trends Neurosci 21:254-259

Heuer HW, Britten KH (2004) Optic flow signals in extrastriate area MST: comparison of perceptual and neuronal sensitivity. J Neurophysiol 91:1314-1326

Huerta MF, Kaas JH (1990) Supplementary eye field as defined by intracortical microstimulation: connections in macaques. J Comp Neurol 293:299-330

Ilg UJ, Schumann S, Thier P (2004) Posterior parietal cortex neurons encode target motion in world-centered coordinates. Neuron 43:145-151

Kawawaki D, Shibata T, Goda N, Doya K, Kawato M (2006) Anterior and superior lateral occipito-temporal cortex responsible for target motion prediction during overt and covert visual pursuit. Neurosci Res 54:112-123

Komatsu H, Wurtz RH (1988a) Relation of cortical areas MT and MST to pursuit eye movements. I. Localization and visual properties of neurons. J Neurophysiol 60:580-603 
Komatsu H, Wurtz RH (1988b) Relation of cortical areas MT and MST to pursuit eye movements. III. Interaction with full-field visual stimulation. J Neurophysiol 60:621-644

Kurkin S, Shichinohe N, Akao T, Fukushima J, Kikuro Fukushima K (2009) MST activity during memory-based smooth pursuit eye movements: comparison with the supplementary eye fields (SEF). Program No. 559.8. Abstract viewer/itinerary planner. Chicago (IL): Society for Neuroscience, Washington

Leigh RJ, Zee DS (2006) The neurology of eye movements, 4th edn. Oxford University Press, New York

Liu S, Angelaki DE (2009) Vestibular signals in macaque extrastriate visual cortex are functionally appropriate for heading perception. J Neurosci 29:8936-8945

Newsome WT, Pare EB (1988) A selective impairment of motion perception following lesions of the middle temporal visual area (MT). J Neurosci 8:2201-2211
Newsome WT, Wurtz RH, Komatsu H (1988) Relation of cortical areas MT and MST to pursuit eye movements. II. Differentiation of retinal from extraretinal inputs. J Neurophysiol 60:604-620

Recanzone GH, Wurtz RH (2000) Effects of attention on MT and MST neuronal activity during pursuit initiation. J Neurophysiol 83:779-790

Saito H, Yukie M, Tanaka K, Hikosaka K, Fukuda Y, Iwai E (1986) Integration of direction signals of image motion in the superior temporal sulcus of the macaque monkey. J Neurosci 6:145-157

Shichinohe N, Akao T, Kurkin S, Fukushima J, Kaneko CRS, Fukushima K (2009) Memory and decision-making in the frontal cortex during visual motion-processing for smooth pursuit eye movements. Neuron 62:717-732

Thier P, Erickson RG (1992) Responses of visual-tracking neurons from cortical area MST-1 to visual, eye and head motion. Eur J Neurosci 4:539-553 